OPEN ACCESS

Edited by:

Honglin Li,

East China University of Science and Technology, China

Reviewed by:

Pei Hao,

Institut Pasteur of Shanghai (CAS),

China

Zhi-Liang Ji,

Xiamen University, China

*Correspondence:

Honghuang Lin

hhlin@bu.edu

Michael M. Mendelson

Michael.Mendelson@cardio.

chboston.org

Specialty section:

This article was submitted to Experimental Pharmacology and Drug

Discovery,

a section of the journal

Frontiers in Pharmacology

Received: 03 January 2018

Accepted: 23 February 2018

Published: 24 April 2018

Citation:

Mendelson MM, Johannes R, Liu C

Huan T, Yao C, Miao $X$

Murabito JM, Dupuis J, Levy D,

Benjamin EJ and Lin H (2018)

Epigenome-Wide Association Study

of Soluble Tumor Necrosis Factor Receptor 2 Levels in the Framingham

Heart Study.

Front. Pharmacol. 9:207.

doi: 10.3389/fphar.2018.00207

\section{Epigenome-Wide Association Study of Soluble Tumor Necrosis Factor Receptor 2 Levels in the Framingham Heart Study}

\author{
Michael M. Mendelson 1,2,3*, Roby Johannes ${ }^{4}$, Chunyu Liu' ${ }^{1,5}$, Tianxiao Huan ${ }^{1,3}$, \\ Chen Yao ${ }^{1,3}$, Xiao Miao ${ }^{6}$, Joanne M. Murabito ${ }^{1,7}$, Josée Dupuis ${ }^{1,5}$, Daniel Levy ${ }^{1,3}$, \\ Emelia J. Benjamin ${ }^{1,8,9}$ and Honghuang Lin ${ }^{1,10 *}$
}

\begin{abstract}
'National Heart, Lung, and Blood Institute's and Boston University's Framingham Heart Study, Framingham, MA, United States, ${ }^{2}$ Department of Cardiology, Boston Children's Hospital, Boston, MA, United States, ${ }^{3}$ Population Sciences Branch, Division of Intramural Research, National Heart, Lung, and Blood Institute, Bethesda, MD, United States,

${ }^{4}$ Hebrew SeniorLife, Harvard Medical School, Boston, MA, United States, ${ }^{5}$ Department of Biostatistics, Boston University School of Public Health, Boston, MA, United States, ${ }^{6}$ Innovation Research Institute of Traditional Chinese Medicine, Shanghai University of Traditional Chinese Medicine, Shanghai, China, ${ }^{7}$ Section of General Internal Medicine, Department of Medicine, Boston University School of Medicine, Boston, MA, United States, ${ }^{8}$ Section of Cardiovascular Medicine and Preventive Medicine, Department of Medicine, Boston University School of Medicine, Boston, MA, United States, ${ }^{9}$ Department of Epidemiology, Boston University School of Public Health, Boston, MA, United States, ${ }^{10}$ Section of Computational Biomedicine, Department of Medicine, Boston University School of Medicine, Boston, MA, United States
\end{abstract}

Background: Transmembrane tumor necrosis factor (TNF) receptors are involved in inflammatory, apoptotic, and proliferative processes. In the bloodstream, soluble TNF receptor II (STNFR2) can modify the inflammatory response of immune cells and is predictive of cardiovascular disease risk. We hypothesize that sTNFR2 is associated with epigenetic modifications of circulating leukocytes, which may relate to the pathophysiology underlying atherogenic risk.

Methods: We conducted an epigenome-wide association study of sTNFR2 levels in the Framingham Heart Study Offspring cohort (examination 8; 2005-2008). sTNFR2 was quantitated by enzyme immunoassay and DNA methylation by microarray. The concentration of STNFR2 was $\log _{e}$-transformed and outliers were excluded. We conducted linear mixed effects models to test the association between sTNFR2 level and methylation at over 400,000 CpGs, adjusting for age, sex, BMI, smoking, imputed cell count, technical covariates, and accounting for familial relatedness.

Results: The study sample included 2468 participants (mean age: $67 \pm 9$ years, 52\% women, mean sTNFR2 level $2661 \pm 1078 \mathrm{pg} / \mathrm{ml})$. After accounting for multiple testing, we identified $168 \mathrm{CpGs}\left(P<1.2 \times 10^{-7}\right)$ that were differentially methylated in relation to sTNFR2. A substantial proportion (27 CpGs; 16\%) are in the major histocompatibility complex region and in loci overrepresented for antigen binding molecular functions $\left(P=1.7 \times 10^{-4}\right)$ and antigen processing and presentation biological processes $\left(P=1.3 \times 10^{-8}\right)$. Identified $\mathrm{CpGs}$ are enriched in active regulatory regions and associated with expression of 48 cis-genes $( \pm 500 \mathrm{~kb})$ in whole blood $\left(P<1.1 \times 10^{-5}\right)$ that coincide with genes identified in GWAS of diseases of immune dysregulation (inflammatory bowel disease, type 1 diabetes, IgA nephropathy). 
Conclusion: Differentially methylated loci in leukocytes associated with sTNF2 levels reside in active regulatory regions, are overrepresented in antigen processes, and are linked to inflammatory diseases.

Keywords: DNA methylation, TNFR2 levels, inflammation, association studies, epigenome

\section{INTRODUCTION}

Soluble tumor necrosis factor receptor II (sTNFR2) levels are associated with an extensive and diverse range of human diseases (Straczkowski et al., 2002; Turan et al., 2008; DoganavsargilBaysal et al., 2013). In addition, sTNFR2 is a marker of cardiovascular disease (CVD) risk in people with diabetes (Shai et al., 2005) and chronic kidney disease (Neirynck et al., 2015). Tumor necrosis factor receptor II binds to tumor necrosis factors to form a heterocomplex that mediates an intricate inflammatory cascade. Released into the bloodstream, sTNFR2 can modify the inflammatory response of immune cells. A greater understanding of the changes that occur in circulating leukocytes in relation to sTNFR2 levels may lead to improved mechanistic insights, enhanced disease predictive modeling, and highlight novel candidates for the development of therapeutics for CVD and inflammatory-related diseases.

DNA methylation is an epigenetic modification that influences gene expression without changing the underlying genetic code. Adding a methyl group to the cytosine of cytosine-phosphateguanine dinucleotides (CpGs) can modify the binding of transcription factors, influence the conformational structure of chromatin, and thus suppress the transcription of specific genes. Altered DNA methylation has been found to be linked to a variety of neoplastic, inflammatory, cardiometabolic, and atherosclerotic diseases (Ying et al., 2000; Maier and Olek, 2002; Fitzpatrick and Wilson, 2003; Hiltunen and Yla-Herttuala, 2003; GallouKabani and Junien, 2005; Ling and Groop, 2009; Lund and Zaina, 2009).

In order to characterize the potential epigenetic link between sTNFR2 levels and disease, we conducted the first epigenome-wide association study of sTNFR2 levels with DNA methylation in circulating leukocytes from participants in a large community based cohort, the Framingham Heart Study (FHS). We also examined the association of sTNFR2-related differential methylation with gene expression in the same cohort. Finally, we integrated the findings with known biological pathways, genomic links to disease from genome-wide association studies, and targets of known drugs and compounds.

\section{MATERIALS AND METHODS}

\section{Study Sample}

The FHS is a longitudinal community-based cohort, started in 1948. Three generations of participants have been recruited. The present study was focused on participants from the second generation, the Offspring cohort (Kannel et al., 1979) who attended the eighth examination between 2005 and 2008. We excluded participants with self-reported auto-immune diseases, such as rheumatoid arthritis, or who took immune modulating agents (e.g., methotrexate), steroids, and synthetic estrogens. All participants gave written informed consent and the study was approved by the Institutional Review Board of Boston Medical Center.

\section{Measurement of sTNFR2 Levels in Plasma}

Fasting morning blood samples were obtained during the routine clinic visit. The samples were then frozen at $-80^{\circ} \mathrm{C}$ before processing. The sTNFR2 levels were measured by the quantitative enzyme-linked immunosorbent assay per the manufacturers' protocols (R\&D Systems, Minneapolis, MN, United States). The assay shows high reproducibility rate with intra-assay coefficients of variation of $2.3 \%$ (Jefferson et al., 2007). The sTNFR2 levels were natural log transformed and values outside of four standard deviation of the mean were excluded from the analysis $(n=1)$.

\section{DNA Methylation Quantification}

DNA from whole blood samples used for methylation assays was collected at the same examination assessment as the sTNFR2 levels, anthropometric and covariate measurements. Buffy coat preparations were obtained from peripheral whole blood samples and DNA was extracted using the Gentra Puregene DNA extraction kit (Qiagen, Venlo, Netherlands) and then underwent bisulfite conversion using the EZ DNA Methylation kit (Zymo Research, Irvine, CA, United States). Samples underwent whole genome amplification, fragmentation, array hybridization, and single-base pair extension. DNA methylation arrays were run in two laboratory batches at the Johns Hopkins Center for Inherited Disease Research and University of Minnesota Biomedical Genomics Center. The first batch included 576 samples from an earlier CVD case-control study (Joehanes et al., 2013) and the second batch included 2270 samples from the remainder of the offspring cohort participants. DNA methylation results underwent normalization within laboratory batches using the DASEN methodology implemented in the wateRmelon package (Pidsley et al., 2013) in R (version 3.0.2). We excluded samples with missing rate $>1 \%$, poor single nucleotide polymorphism (SNP) matching to the 65 SNP control probe locations, and outliers by multi-dimensional scaling techniques. We also excluded probes with missing rate $>20 \%$ and those previously identified to map to multiple locations (Chen et al., 2013) or to have an underlying SNP (minor allele frequency $>5 \%$ in European participants from the 1000 Genomes Project) at the CpG site, or within $10 \mathrm{bp}$ of the single base extension $(n=42,251)$. The methylation data are available at $\mathrm{dbGaP}$ under the accession number phs000724.v2.p9. 


\section{Gene Expression Assay}

The details of gene expression profiling were previously described (Joehanes et al., 2013). In brief, gene expression was obtained from whole blood samples collected in PAXgene blood tubes (PreAnalytiX, Hombrechtikon, Switzerland) at the same time as DNA methylation samples. RNA was extracted using a whole blood RNA System Kit (QIAGEN, Hilden, Germany) and assayed using the Affymetrix Human Exon Array ST-1.0 (Affymetrix, Santa Clara, CA, United States). Robust Multi-array Average (RMA) package in $\mathrm{R}$ was used to normalize gene expression values. Residuals were obtained from linear regression models after adjustment for technical covariates and imputed differential blood cell proportions. The pedigreemm package was used to remove the variation due to age, sex, and family structure. The gene expression data is available at dbGaP under the accession number phs000363.v16.p10.

\section{Clinical Covariate Assessment}

Height and weight were measured using established protocols and body mass index (BMI) was calculated as weight (in kilograms) divided by height (in meters) squared. Self-reported cigarette smoking was categorized based on current smoking status (current smoker, non-current smoker).

\section{Statistical Analyses}

\section{Epigenome-Wide Association Study of sTNFR2}

We used linear mixed effects regression models, whereas the untransformed beta value of methylation at the CpG site was treated as the dependent variable, and the sTNFR2 level was treated as the independent variable, adjusted for age, sex, BMI, smoking status, and technical covariates. The sex was coded as " 1 " for men and " 2 " for women. For the smoking status, we coded current smokers as " 1 " and the rest of participants as "0." Technical covariates included known batch effects (methylation array, row, and column) and two methylation principal components to account for unmeasured batch effects. To account for the family relatedness in Framingham, a covariance matrix was calculated based on the pedigree, and was used as the random effect for the linear mixed effects models. We additionally adjusted for the proportion of six cell types imputed via a reference panel (Houseman method) (Houseman et al., 2012), including $\mathrm{CD}^{+} \mathrm{T}$ cells, $\mathrm{CD} 4^{+} \mathrm{T}$ cell, natural killer cells, B cells, monocytes and granulocytes. Bonferroni correction was used to adjust for multiple testing, and significant CpG sites were defined as those with $P<0.05 / \mathrm{N}$, where $\mathrm{N}$ was the number of tests. The analyses were performed using the "lmekin" function within R package "kinship", and the clinical and technical covariates were used as fixed effect in the models.

\section{Association of sTNFR2-Related CpG Methylation With Gene Expression}

Each epigenome-wide significant CpG from the sTNFR2 EWAS analysis was tested using linear mixed effect models for the association with expression levels of genes in cis $( \pm 500 \mathrm{~kb})$. The cis expression-methylation association models specified the
TABLE 1 | Clinical characteristics of studied samples.

\begin{tabular}{ll}
\hline Characteristics & Total $(\boldsymbol{n}=\mathbf{2 4 6 8})$ \\
\hline Women, $n(\%)$ & $1282(51.9 \%)$ \\
Age, year $\pm S D$ & $67 \pm 9$ \\
sTNFR2 levels, pg/ml & $2661 \pm 1078$ \\
Cigarette smoking, $\mathrm{n}(\%)$ & $217(8.8 \%)$ \\
Body mass index, $\mathrm{kg} / \mathrm{m}^{2}$ & $28.3 \pm 5.3$ \\
\hline
\end{tabular}

gene expression residual as the dependent variable, the DNA methylation residual as the independent variable of interest and additionally adjusted for 25 methylation surrogate variables and 25 expression surrogate variables to account for unmeasured technical and batch effects. Multiple testing was accounted for by Bonferroni correction for the number of $\mathrm{CpGs}$ in distinct loci $(>500 \mathrm{~kb}$ ) tested times the number of unique cis genes on the expression array.

\section{Enrichment Analysis}

We used eFORGE (Breeze et al., 2016) (v1.2) ${ }^{1}$ to examine if sTNFR2-related CpG sites are enriched in DNAse I hypersensitivity hotspots (markers of active regulatory regions) and loci with overlapping histone modifications ( $\mathrm{H} 3 \mathrm{Kmel}$, H3Kme4, H3K9me3, H3K27me3, and H3K36me3) across cell lines and tissues from the Roadmap Epigenome, BLUEPRINT Epigenome, and ENCODE consortia data (Adams et al., 2012; ENCODE Project Consortium et al., 2012; Roadmap Epigenomics Consortium et al., 2015).

We used the PANTHER Overrepresentation Test ${ }^{2}$ (release 20171205) to identify whether identified gene loci are overrepresented in specific molecular function and biological process gene ontologies (slim version) with Fisher's Exact test and false discovery rate (FDR) multiple testing correction. We then used FUMA (Watanabe et al., 2017) (v1.2.4) ${ }^{3}$ to examine whether CpG-related differentially expressed genes are enriched for specific diseases identified in genome-wide association studies (GWAS) from GWAS catalog version e85 (2016.09.27), restricting to at least three overlapping genes, and FDR multiple testing correction.

\section{RESULTS}

The study sample, outlined in Table 1, included 2,468 FHS participants (mean age $67 \pm 9$ years, 52\% women) from the eighth examination cycle (2005-2008). The distribution of sTNFR2 levels is shown in Supplementary Figure 1 (mean level $2661 \pm 1078 \mathrm{pg} / \mathrm{ml})$.

\section{Association of Methylation Profiles With sTNFR2 Levels}

We found methylation at $168 \mathrm{CpG}$ sites to be associated at epigenome-wide thresholds $\left(P<1.2 \times 10^{-7}\right)$ with sTNFR2 levels

\footnotetext{
${ }^{1}$ eforge.cs.ucl.ac.uk

${ }^{2}$ www.pantherdb.org

${ }^{3}$ fuma.ctglab.nl
} 


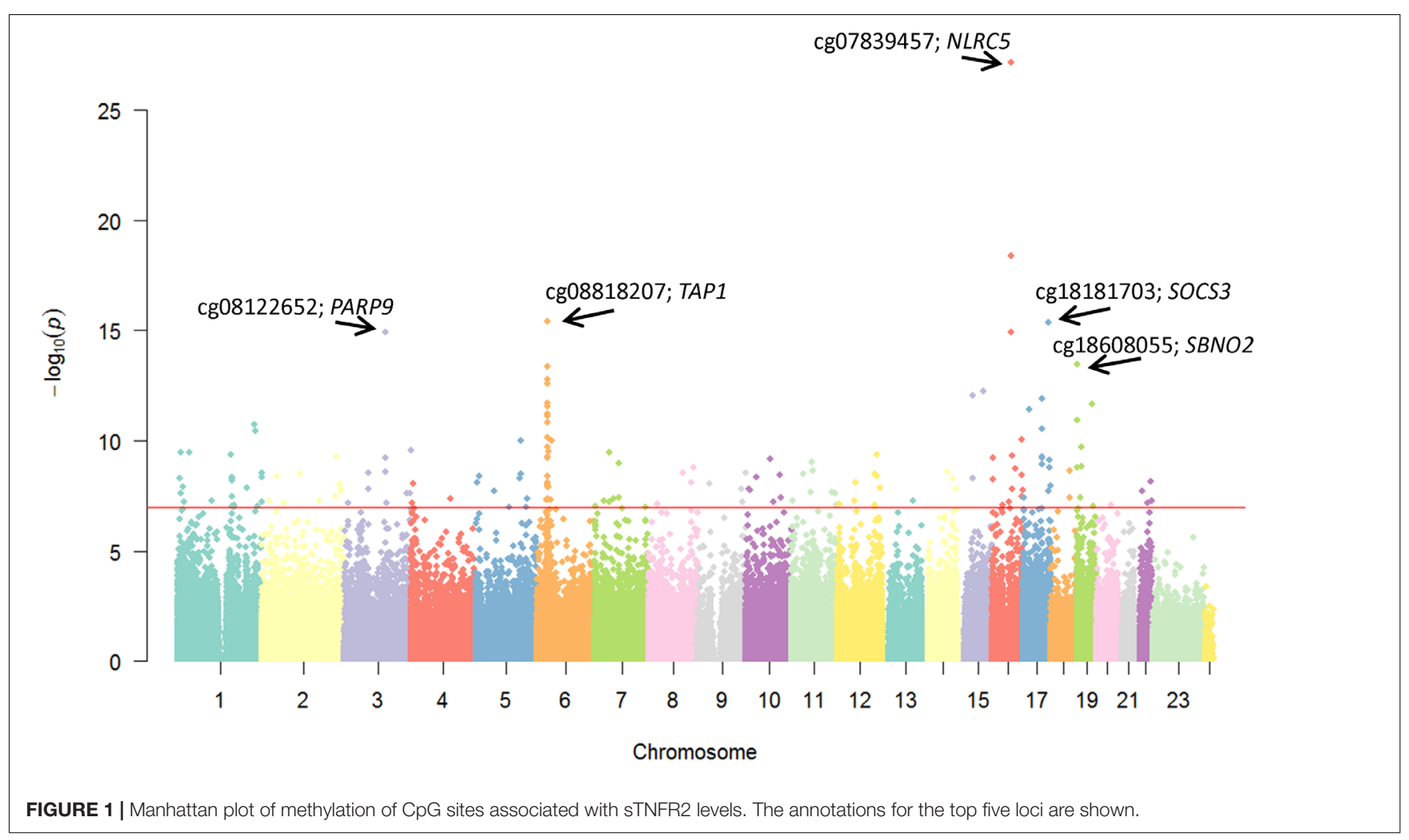

(complete list in Supplementary Table 1) after adjustment for age, sex, BMI, smoking, imputed cell counts, methylation principal components, technical covariates, and accounting for familial relatedness. Figure 1 depicts the Manhattan plot of CpG sites associated with sTNFR2 with the top CpG sites noted in Table 2. The strongest association was at $\operatorname{cg} 07839457\left(P=3.2 \times 10^{-22}\right)$, located 436 bp upstream of the transcription start site of NLRC5, which is involved in cytokine response, inhibition of NF-кB activation and negative regulation of type I interferon signaling pathways (Cui et al., 2010).

\section{Functional and Regulatory Annotation of sTNFR2-Related CpG Sites}

The 168 sTNFR2-related differentially methylated CpGs are enriched in active regulatory regions (DHS hotspots) across most blood cell lines (especially inflammatory macrophages, $\left.P<1 \times 10^{-15}\right)$, but also vascular and cardiac tissues $\left(P<1 \times 10^{-4}\right)$ (Supplementary Figure 2). Many sTNFR2-related CpGs are located within histone modification regions; primarily H3K4me1 (marker of enhancers), and to a lesser extent H3K4me3 (marker of promoters) and H3K36me3 (found in actively transcribed gene bodies) in blood cell lines (Supplementary Table 3). Unsurprisingly, a large proportion of sTNFR2-related CpG sites are located within the major histocompatibility complex (MHC) region (27 CpGs; 16\%). Other top loci were in gene regions known to be involved in NF- $\mathrm{B}$ and cytokine pathways, such as NLRC5, SOCS3, BCL3 and SBNO2. sTNFR2related loci are overrepresented in antigen binding molecular functions $\left(P=1.7 \times 10^{-4}, \mathrm{FDR}=3.2 \times 10^{-2}\right)$ and antigen processing and presentation biological processes $\left(P=1.3 \times 10^{-8}\right.$, $\mathrm{FDR}=3.1 \times 10^{-6}$ ).

\section{Association of sTNFR2-Related CpG Sites With Gene Expression}

We also examined if the sTNFR2-related CpG sites are associated with gene expression in whole blood. The $168 \mathrm{CpG}$ sites are in 127 distinct loci, comprising 4352 genes in cis ( $\pm 500 \mathrm{~kb})$. As shown in Supplementary Table 2, these CpGs were associated with the expression of 27 genes after adjustment for multiple testing $\left(P<1.1 \times 10^{-5}\right)$. Over $5 \%$ of the variation in expression (partial $\mathrm{r}^{2}$ ) was explained by methylation at the identified CpGs for three genes (HCP5, AIM2, and NMUR1). HCP5 is a non-coding gene located in the MHC class I region, and is linked to viral interactions, neoplastic progression, and psoriatic arthritis (Liu et al., 2008). The expression of AIM2 is induced by interferon gamma and plays a role in the innate immune system. NMUR1 is a transmembrane protein involved in signal transduction.

We then assessed the disease relevance of the 27 genes that demonstrated differential expression with sTNFR2-related CpG sites. Table 3 shows the top enriched gene sets. Many of them are involved in immune dysregulation (inflammatory bowel disease, type 1 diabetes, and autoimmune thyroid disease), clotting factors (fibrinogen), and obesity-related traits. A query of the DrugBank database (version 5.0.9) (Law et al., 2014) showed that six of these genes were targets of known drugs or compounds, including PSMB8 (carfilzomib), S100P (chromolyn sodium), TYMP (tipiracil, cidofovir, trifuridine, fluorouracil, floxuridine, 
TABLE 2 | Top 20 CpG sites associated with sTNFR2 levels.

\begin{tabular}{|c|c|c|c|c|c|c|}
\hline CPG & Chr & Position (hg19) & Closest gene & Effect & SE & $P$-value \\
\hline cg07839457 & 16 & $57,023,022$ & NLRC5 & -0.0520 & 0.0047 & $6.7 \times 10^{-28}$ \\
\hline cg16411857 & 16 & $57,023,191$ & NLRC5 & -0.0246 & 0.0027 & $4.3 \times 10^{-19}$ \\
\hline cg08818207 & 6 & $32,820,355$ & TAP1 & -0.0201 & 0.0025 & $3.9 \times 10^{-16}$ \\
\hline cg18181703 & 17 & $76,354,621$ & socs3 & -0.0217 & 0.0026 & $4.1 \times 10^{-16}$ \\
\hline cg08159663 & 16 & $57,022,486$ & NLRC5 & -0.0148 & 0.0018 & $1.1 \times 10^{-15}$ \\
\hline cg08122652 & 3 & $122,281,939$ & PARP9 & -0.0196 & 0.0024 & $1.2 \times 10^{-15}$ \\
\hline cg18608055 & 19 & $1,130,866$ & SBNO2 & -0.0156 & 0.0020 & $3.4 \times 10^{-14}$ \\
\hline cg08099136 & 6 & $32,811,251$ & PSMB8 & -0.0195 & 0.0026 & $4.4 \times 10^{-14}$ \\
\hline cg01309328 & 6 & $32,811,253$ & PSMB8 & -0.0170 & 0.0023 & $1.7 \times 10^{-13}$ \\
\hline cg07333021 & 6 & $30,612,330$ & C6orf134 & 0.0133 & 0.0018 & $2.6 \times 10^{-13}$ \\
\hline cg23387863 & 15 & $77,472,416$ & SGK269 & -0.0147 & 0.0020 & $5.4 \times 10^{-13}$ \\
\hline cg22107533 & 15 & $45,028,083$ & TRIM69 & -0.0172 & 0.0024 & $9.1 \times 10^{-13}$ \\
\hline cg16936953 & 17 & $57,915,665$ & TMEM49 & -0.0251 & 0.0035 & $1.2 \times 10^{-12}$ \\
\hline cg11187245 & 6 & $31,323,397$ & $H L A-B$ & -0.0209 & 0.0030 & $2.0 \times 10^{-12}$ \\
\hline cg26470501 & 19 & $45,252,955$ & BCL3 & -0.0149 & 0.0021 & $2.2 \times 10^{-12}$ \\
\hline cg16186435 & 6 & $32,810,833$ & PSMB8 & -0.0140 & 0.0020 & $2.7 \times 10^{-12}$ \\
\hline cg02656560 & 17 & $19,967,600$ & SPECC1 & -0.0114 & 0.0016 & $3.7 \times 10^{-12}$ \\
\hline cg13031097 & 6 & $31,322,577$ & $H L A-B$ & -0.0195 & 0.0028 & $6.3 \times 10^{-12}$ \\
\hline cg23533285 & 6 & $31,322,348$ & $H L A-B$ & -0.0180 & 0.0026 & $7.4 \times 10^{-12}$ \\
\hline cg07573872 & 19 & $1,126,342$ & SBNO2 & -0.0192 & 0.0028 & $1.2 \times 10^{-11}$ \\
\hline
\end{tabular}

SE, standard error.

TABLE 3 | Enrichment of identified genes in genome-wide association studies of human disease.

\begin{tabular}{|c|c|c|c|c|c|}
\hline Gene set & $\begin{array}{c}\text { Number of genes } \\
\text { in the set }\end{array}$ & $\begin{array}{c}\text { Number of } \\
\text { overlapping genes }\end{array}$ & $P$-value & $\begin{array}{l}\text { Adjusted } \\
P \text {-value }\end{array}$ & Overlapping genes \\
\hline $\begin{array}{l}\text { Type } 1 \text { diabetes and } \\
\text { autoimmune thyroid diseases }\end{array}$ & 23 & 3 & $4.4 \times 10^{-9}$ & $4.8 \times 10^{-6}$ & HCP5, PRRC2A, TAP2 \\
\hline Fibrinogen & 41 & 3 & $5.0 \times 10^{-8}$ & $1.8 \times 10^{-5}$ & CPT1B, SLC22A4, SLC22A5 \\
\hline Ulcerative colitis & 447 & 5 & $2.4 \times 10^{-6}$ & $3.2 \times 10^{-4}$ & $\begin{array}{l}\text { LSP1, SLC22A4, SLC22A5, TCF19, } \\
\text { PRRC2A }\end{array}$ \\
\hline Inflammatory bowel disease & 719 & 5 & $3.5 \times 10^{-5}$ & $2.2 \times 10^{-3}$ & $\begin{array}{l}\text { LSP1, SLC22A4, SLC22A5, TCF19, } \\
\text { PRRC2A }\end{array}$ \\
\hline Obesity-related traits & 745 & 4 & $4.6 \times 10^{-4}$ & $1.2 \times 10^{-2}$ & IFI16, AIM2, ODF3B, S100P \\
\hline Blood protein levels & 914 & 4 & $1.2 \times 10^{-3}$ & $2.4 \times 10^{-2}$ & HCP5, PRRC2A, TAP2, TAP2, PSMB8 \\
\hline Crohn's disease & 612 & 3 & $2.0 \times 10^{-3}$ & $3.6 \times 10^{-2}$ & SLC22A4, SLC22A5, HLA-F \\
\hline
\end{tabular}

and capecitabine), GLTP (capric acid, lauric acid, oleic acid, and sphingosine), SLC22A4 and SLC22A5 (by numerous drugs that influence transmembrane channels).

\section{DISCUSSION}

We present the first epigenome-wide analysis of leukocytederived DNA methylation in relation to sTNFR2 levels in a large community-based cohort. We identified differential methylation at $168 \mathrm{CpGs}$ within 127 distinct genomic loci that are enriched for active regulatory regions across inflammatory macrophages and vascular tissues. Our results highlight functional relevance of genomic regulation of antigen presentation and processing in relation to sTNFR2 levels with multiple loci identified in the MHC region. Differentially methylated loci are associated with gene expression of 27 cis-genes in whole blood that have clinical relevance for inflammatory diseases, clotting factors, and obesityrelated traits. Taken together, delineation of genomic regulatory alterations in relation to sTNFR2 demonstrates a host of altered pathways that may be important targets for novel or known therapeutics to prevent CVD and other sTNFR2-related diseases.

The most significant $\mathrm{CpG}$ site resides within the promoter region of NLRC5. The NLRC5 gene encodes a nucleotide binding protein that belongs to the highly conserved NOD-like protein family, which regulates multiple interferon signaling pathways (Cui et al., 2010). NLRC5 has been shown to be cardioprotective; deficiency of NLRC5 exacerbates high fat diet-induced heart injury in murine models (Ma and Xie, 2017). Another significant sTNFR2-related CpG site, cg08818207, is in the first intron of TAP1, which encodes a transporter belonging to the ATP binding cassette subfamily. The protein is involved in the transportation of a variety of molecules across the cell membranes. It is essential to the presentation of peptides to human leukocyte antigen 
molecules. Mutations in TAP1 result in immune-related diseases such as MHC class I deficiency (Hanalioglu et al., 2017) but are also associated with cardiometabolic diseases, such as type 2 diabetes (Li et al., 2014).

As expected, among genes that showed differential expression with sTNFR2-related CpG sites, many of them are involved in inflammatory and autoimmune diseases. One example is SLC22A4, which encodes an organic cation transporter that plays an essential role in the elimination of small organic cations such as environmental toxins and drugs (Morrissey et al., 2013; Nigam et al., 2015). It has already been proposed as potential drug target for the treatment of inflammatory diseases such as rheumatoid arthritis (Tokuhiro et al., 2003; Martinez et al., 2006; Maeda et al., 2007) and Crohn's disease (Leung et al., 2006). An additional five identified genes are targets of known drugs or compounds. For example, carfilzomib, an anti-neoplastic and selective proteasome inhibitor, influences PSMB8, proteasome subunit beta 8 . Carfilzomib has already been shown to have cardiovascular effects with substantial cardiotoxicity observed in clinical oncology trials (Waxman et al., 2017).

Our study has limitations. First, unlike genetic sequence variants, differences in DNA methylation may occur secondary to the phenotype of interest and not causal effects. Despite not being able to determine the direction of effect, secondary changes may still be important for downstream consequences, such as CVD. Second, current study measured DNA methylation profiles in the whole blood, which contains a mixture of different types of cells. Although we adjusted for cell proportion changes using statistical approaches, residual confounding due to unmeasured cell type differences or other unmeasured technical or clinical factors may be present. Third, although we assayed over four hundred thousand CpGs, there are many unmeasured CpGs sites and therefore additional methylation differences may be relevant. Fourth, we only assessed DNA methylation from leukocytes and did not assay DNA methylation concurrently in other tissues in the body where findings may differ. Therefore, our assessment is focused on circulating immune-cell related changes. Fifth, as sTNFR2 is not a widely measured biomarker, we were unable to replicate our findings in an external cohort with DNA methylation. Further studies are needed to determine if our findings replicate in other cohorts and are generalizable to other age groups and ethnicities/races.

\section{CONCLUSION}

EWAS of leukocyte DNA for CVD-related inflammatory biomarkers represents a powerful approach to gain novel

\section{REFERENCES}

Adams, D., Altucci, L., Antonarakis, S. E., Ballesteros, J., Beck, S., Bird, A., et al. (2012). BLUEPRINT to decode the epigenetic signature written in blood. Nat. Biotechnol. 30, 224-226. doi: 10.1038/nbt.2153

Breeze, C. E., Paul, D. S., Van Dongen, J., Butcher, L. M., Ambrose, J. C., Barrett, J. E., et al. (2016). eFORGE: a tool for identifying cell type-specific signal in epigenomic data. Cell Rep. 17, 2137-2150. doi: 10.1016/j.celrep.2016.10.059 insights into affected biological pathways. In our sTNFR2 EWAS, we highlight antigen processing pathways and specific genes in NF- $\kappa \mathrm{B}$ signaling and cytokine development and release. Genes in these pathways have previously been linked to human disease GWAS of inflammatory diseases, clotting factors, and obesity-related traits. Further experimental studies and trials are needed to determine if perturbation of these genes will support the repurposing of known drugs or development of novel compounds which may prevent or treat CVD and other sTNFR2 related diseases.

\section{AUTHOR CONTRIBUTIONS}

MMM and HL drafted the manuscript. MMM, RJ, CL, TH, CY, and HL performed the analyses. XM, JMM, JD, DL, and EJB critically reviewed the manuscript.

\section{FUNDING}

This work is supported by NIH grants R56AG029451 (JM), 5R01HL092577 (EB), and 5R01HL128914 (EB). Measurement of inflammatory biomarkers was funded through R01 HL064753 and R01 HL076784 and further supported by 1R01 HL64753 (EB), R01AG028321 (EB). Additional support was from the NHLBI K99 HL136875 (MM), Boston University Digital Health Initiative (HL), and the National Center for Advancing Translational Sciences, National Institutes of Health, through BU-CTSI Grant Number 1UL1TR001430. The laboratory work for this investigation was funded by the Division of Intramural Research, National Heart, Lung, and Blood Institute, National Institutes of Health, and by a Director's Challenge Award, National Institutes of Health (DL). The Framingham Heart Study is funded by National Institutes of Health Contract N01-HC-25195; HHSN268201500001I. The views expressed in this manuscript are those of the authors and do not necessarily represent the views of the National Heart, Lung, and Blood Institute, the National Institutes of Health, or the U.S. Department of Health and Human Services.

\section{SUPPLEMENTARY MATERIAL}

The Supplementary Material for this article can be found online at: https://www.frontiersin.org/articles/10.3389/fphar. 2018.00207/full\#supplementary-material

Chen, Y. A., Lemire, M., Choufani, S., Butcher, D. T., Grafodatskaya, D., Zanke, B. W., et al. (2013). Discovery of cross-reactive probes and polymorphic CpGs in the Illumina Infinium HumanMethylation450 microarray. Epigenetics 8, 203-209. doi: 10.4161/epi.23470

Cui, J., Zhu, L., Xia, X., Wang, H. Y., Legras, X., Hong, J., et al. (2010). NLRC5 negatively regulates the NF-кB and type I interferon signaling pathways. Cell 141, 483-496. doi: 10.1016/j.cell.2010. 03.040 
Doganavsargil-Baysal, O., Cinemre, B., Aksoy, U. M., Akbas, H., Metin, O., Fettahoglu, C., et al. (2013). Levels of TNF-alpha, soluble TNF receptors (sTNFR1, sTNFR2), and cognition in bipolar disorder. Hum. Psychopharmacol. 28, 160-167. doi: 10.1002/hup.2301

ENCODE Project Consortium, Dunham, I., Kundaje, A., Aldred, S. F., Collins, P. J., Davis, C. A., et al. (2012). An integrated encyclopedia of DNA elements in the human genome. Nature 489, 57-74. doi: 10.1038/nature11247

Fitzpatrick, D. R., and Wilson, C. B. (2003). Methylation and demethylation in the regulation of genes, cells, and responses in the immune system. Clin. Immunol. 109, 37-45. doi: 10.1016/S1521-6616(03)00205-5

Gallou-Kabani, C., and Junien, C. (2005). Nutritional epigenomics of metabolic syndrome: new perspective against the epidemic. Diabetes 54, 1899-1906. doi: 10.2337/diabetes.54.7.1899

Hanalioglu, D., Ayvaz, D. C., Ozgur, T. T., Van Der Burg, M., Sanal, O., and Tezcan, I. (2017). A novel mutation in TAP1 gene leading to MHC class I deficiency: report of two cases and review of the literature. Clin. Immunol. 178, 74-78. doi: 10.1016/j.clim.2017.01.011

Hiltunen, M. O., and Yla-Herttuala, S. (2003). DNA methylation, smooth muscle cells, and atherogenesis. Arterioscler. Thromb. Vasc. Biol. 23, 1750-1753. doi: 10.1161/01.ATV.0000092871.30563.41

Houseman, E. A., Accomando, W. P., Koestler, D. C., Christensen, B. C., Marsit, C. J., Nelson, H. H., et al. (2012). DNA methylation arrays as surrogate measures of cell mixture distribution. BMC Bioinform. 13:86. doi: 10.1186/1471-21 05-13-86

Jefferson, A. L., Massaro, J. M., Wolf, P. A., Seshadri, S., Au, R., Vasan, R. S., et al. (2007). Inflammatory biomarkers are associated with total brain volume: the Framingham Heart Study. Neurology 68, 1032-1038. doi: 10.1212/01.wnl. 0000257815.20548.df

Joehanes, R., Ying, S., Huan, T., Johnson, A. D., Raghavachari, N., Wang, R., et al. (2013). Gene expression signatures of coronary heart disease. Arterioscler. Thromb. Vasc. Biol. 33, 1418-1426. doi: 10.1161/ATVBAHA.112. 301169

Kannel, W. B., Feinleib, M., Mcnamara, P. M., Garrison, R. J., and Castelli, W. P. (1979). An investigation of coronary heart disease in families. The Framingham offspring study. Am. J. Epidemiol. 110, 281-290. doi: 10.1093/oxfordjournals. aje.a1 12813

Law, V., Knox, C., Djoumbou, Y., Jewison, T., Guo, A. C., Liu, Y., et al. (2014). DrugBank 4.0: shedding new light on drug metabolism. Nucleic Acids Res. 42, D1091-D1097. doi: 10.1093/nar/gkt1068

Leung, E., Hong, J., Fraser, A. G., Merriman, T. R., Vishnu, P., and Krissansen, G. W. (2006). Polymorphisms in the organic cation transporter genes SLC22A4 and SLC22A5 and Crohn's disease in a New Zealand Caucasian cohort. Immunol. Cell Biol. 84, 233-236. doi: 10.1111/j.1440-1711.2006.01423.x

Li, Y. Y., Gao, W., Pang, S. S., Min, X. Y., Yang, Z. J., Wang, H., et al. (2014). TAP1 I333V gene polymorphism and type 1 diabetes mellitus: a meta-analysis of 2248 cases. J. Cell Mol. Med. 18, 929-937. doi: 10.1111/jcmm.12244

Ling, C., and Groop, L. (2009). Epigenetics: a molecular link between environmental factors and type 2 diabetes. Diabetes 58, 2718-2725. doi: 10. 2337/db09- 1003

Liu, Y., Helms, C., Liao, W., Zaba, L. C., Duan, S., Gardner, J., et al. (2008). A genome-wide association study of psoriasis and psoriatic arthritis identifies new disease loci. PLoS Genet. 4:e1000041. doi: 10.1371/journal.pgen.1000041

Lund, G., and Zaina, S. (2009). Atherosclerosis risk factors can impose aberrant DNA methylation patterns: a tale of traffic and homocysteine. Curr. Opin. Lipidol. 20, 448-449. doi: 10.1097/MOL.0b013e3283309928

Ma, S. R., and Xie, X. W. (2017). NLRC5 deficiency promotes myocardial damage induced by high fat diet in mice through activating TLR4/NFкВ. Biomed. Pharmacother. 91, 755-766. doi: 10.1016/j.biopha.2017. 03.062

Maeda, T., Hirayama, M., Kobayashi, D., Miyazawa, K., and Tamai, I. (2007). Mechanism of the regulation of organic cation/carnitine transporter 1 (SLC22A4) by rheumatoid arthritis-associated transcriptional factor RUNX1 and inflammatory cytokines. Drug Metab. Dispos. 35, 394-401. doi: 10.1124/ dmd.106.012112
Maier, S., and Olek, A. (2002). Diabetes: a candidate disease for efficient DNA methylation profiling. J. Nutr. 132(Suppl. 8), 2440S-2443S. doi: 10.1093/jn/132. 8.2440 S

Martinez, A., Valdivia, A., Pascual-Salcedo, D., Balsa, A., Fernandez-Gutierrez, B., De La Concha, E., et al. (2006). Role of SLC22A4, SLC22A5, and RUNX1 genes in rheumatoid arthritis. J. Rheumatol. 33, 842-846. doi: 10.1016/j.clim.2006. 04.114

Morrissey, K. M., Stocker, S. L., Wittwer, M. B., Xu, L., and Giacomini, K. M. (2013). Renal transporters in drug development. Annu. Rev. Pharmacol. Toxicol. 53, 503-529. doi: 10.1146/annurev-pharmtox-011112-140317

Neirynck, N., Glorieux, G., Schepers, E., Verbeke, F., and Vanholder, R. (2015). Soluble tumor necrosis factor receptor 1 and 2 predict outcomes in advanced chronic kidney disease: a prospective cohort study. PLoS One 10:e0122073. doi: 10.1371/journal.pone.0122073

Nigam, S. K., Wu, W., Bush, K. T., Hoenig, M. P., Blantz, R. C., and Bhatnagar, V. (2015). Handling of drugs, metabolites, and uremic toxins by kidney proximal tubule drug transporters. Clin. J. Am. Soc. Nephrol. 10, 2039-2049. doi: 10.2215/ CJN.02440314

Pidsley, R., Cc, Y. W., Volta, M., Lunnon, K., Mill, J., and Schalkwyk, L. C. (2013). A data-driven approach to preprocessing Illumina $450 \mathrm{~K}$ methylation array data. BMC Genom. 14:293. doi: 10.1186/1471-2164-14-293

Roadmap Epigenomics Consortium, Kundaje, A., Meuleman, W., Ernst, J., Bilenky, M., Yen, A., et al. (2015). Integrative analysis of 111 reference human epigenomes. Nature 518, 317-330. doi: 10.1038/nature14248

Shai, I., Schulze, M. B., Manson, J. E., Rexrode, K. M., Stampfer, M. J., Mantzoros, C., et al. (2005). A prospective study of soluble tumor necrosis factor-alpha receptor II (sTNF-RII) and risk of coronary heart disease among women with type 2 diabetes. Diabetes Care 28, 1376-1382. doi: 10.2337/diacare. 28.6.1376

Straczkowski, M., Kowalska, I., Stepien, A., Dzienis-Straczkowska, S., Szelachowska, M., and Kinalska, I. (2002). Increased plasma-soluble tumor necrosis factor-alpha receptor 2 level in lean nondiabetic offspring of type 2 diabetic subjects. Diabetes Care 25, 1824-1828. doi: 10.2337/diacare.25.10.1824

Tokuhiro, S., Yamada, R., Chang, X., Suzuki, A., Kochi, Y., Sawada, T., et al. (2003). An intronic SNP in a RUNX1 binding site of SLC22A4, encoding an organic cation transporter, is associated with rheumatoid arthritis. Nat. Genet. 35, 341-348. doi: 10.1038/ng1267

Turan, B., Pfister, K., Diener, P. A., Hell, M., Moller, B., Boyvat, A., et al. (2008). Soluble tumour necrosis factor receptors sTNFR1 and sTNFR2 are produced at sites of inflammation and are markers of arthritis activity in Behcet's disease. Scand. J. Rheumatol. 37, 135-141. doi: 10.1080/03009740701747137

Watanabe, K., Taskesen, E., Van Bochoven, A., and Posthuma, D. (2017). Functional mapping and annotation of genetic associations with FUMA. Nat. Commun. 8:1826. doi: 10.1038/s41467-017-01261-5

Waxman, A. J., Clasen, S., Hwang, W. T., Garfall, A., Vogl, D. T., Carver, J., et al. (2017). Carfilzomib-associated cardiovascular adverse events: a systematic review and meta-analysis. JAMA Oncol. doi: 10.1001/jamaoncol.2017.4519 [Epub ahead of print].

Ying, A. K., Hassanain, H. H., Roos, C. M., Smiraglia, D. J., Issa, J. J., Michler, R. E., et al. (2000). Methylation of the estrogen receptor-alpha gene promoter is selectively increased in proliferating human aortic smooth muscle cells. Cardiovasc. Res. 46, 172-179. doi: 10.1016/S0008-6363(00)00004-3

Conflict of Interest Statement: The authors declare that the research was conducted in the absence of any commercial or financial relationships that could be construed as a potential conflict of interest.

Copyright (C) 2018 Mendelson, Johannes, Liu, Huan, Yao, Miao, Murabito, Dupuis, Levy, Benjamin and Lin. This is an open-access article distributed under the terms of the Creative Commons Attribution License (CC BY). The use, distribution or reproduction in other forums is permitted, provided the original author(s) and the copyright owner are credited and that the original publication in this journal is cited, in accordance with accepted academic practice. No use, distribution or reproduction is permitted which does not comply with these terms. 